\title{
Vom Dilemma der Gesetzgebungslehre in Deutschland
}

\author{
Helmuth Schulze-Fielitz"
}

\begin{abstract}
Rezension zu Florian Schärdel, Die Bücherkodifikation. Untersuchung einer Gesetzgebungstechnik, Baden-Baden (Nomos - Gesetzgebung und Verfassung, Bd. 4) 2012, 257 Seiten, 67.- €. ISBN 978-3-8329-7174-8
\end{abstract}

1. Der vom Autor geprägte Begriff der „Bücherkodifikation“ (S. 22 f.) zielt auf die Entstehung von Kodifikationen, die zeitlich gestreckt über mehrere Legislaturperioden in mehreren Büchern als Teil(gesetz)en einer umfassenden Kodifikation entstehen. Seine Berliner Dissertation hat vor allem die Planung eines Umweltgesetzbuches (UGB) mit seinen vorerst gescheiterten Versuchen zu Teilkodifikationen in Form von Büchern vor Augen, zieht aber immer wieder vergleichend als anderes „Referenzgebiet“ das mittlerweile seit mehr als dreieinhalb Jahrzehnten noch immer unvollendete Projekt eines Sozialgesetzbuches (SGB) heran. Das erkenntnis- und argumentationsleitende Interesse des Autors wird von der Hypothese bestimmt, dass für diese Gesetzgebungstechnik der Bücherkodifikation erfolgversprechende Vorteile bei den Realisierungschancen einer Kodifikation sprechen (vgl. S. 52, 99 f., 104ff., 133), auch wenn diese Vorgehensweise bei dem Projekt eines UGB bislang nicht erfolgreich war (vgl. S. 57ff.).

2. a) Inhaltlich arbeitet Verf. sein Untersuchungsprogramm (S. 24 f.) fast ohne Redundanzen zielführend, mit klaren Problemstellungen und häufigen (etwas oft wiederholenden) Zwischenresultaten, insgesamt freilich wenig spektakulär ab (Ergebnisse der Arbeit: S. 235-243). Die erste Hälfte der Studie gilt Grundsatzproblemen einer Kodifikation. Der einführende narrative Überblick über die Kodifikationsgeschichten im Sozialrecht seit den Anfängen der RVO als erster Kodifikation im modernen Sinne (S. 27ff.) und im Umweltrecht (S. 43ff.) zeigt neben der Gemeinsamkeit des gesetzgebungstechnischen Ansatzes einer Bücherkodifikation wesentliche Unterschiede nach gesetzlicher Ausgangslage, nach der Existenz wissenschaftlicher Vorarbeiten und nach der Verteilung der verfassungsrechtlichen Gesetzgebungskompetenzen. Der Begriff der Kodifikation sei in der deutschen Rechtswissenschaft „mythisch aufgeladen“ (S. 61ff.) - vom Kodifikationsstreit zwischen Thibaut und Savigny als wirkungsmächtiger Erzählung bis hin zum BGB als zentralem Vergleichsgegenstand - mit der Folge, dass eine Bücherkodifikation es neben jenem Idealbild schwer hat: Sie hat anders als z. B. die großen Kodifikationen, mit denen sich nicht selten der Name eines Gesetzesschöpfers verbindet (z. B. „Code Napoleon“) keinen zentralen Stifter, stellt den Anspruch von Kodifikationen auf Dauerhaftigkeit und

* Prof. Dr. Helmuth Schulze-Fielitz war Inhaber des Lehrstuhls für Öffentliches Recht, Umweltrecht und Verwaltungswissenschaften an der Juristischen Fakultät der Bayerischen Julius-Maximilians-Universität Würzburg. 
Lückenlosigkeit, abgeschwächt aber auch auf systematische Rationalität infrage, und sie kann auch sonst kaum eine identitätsstiftende Wirkung entfalten, wie sie einst noch den Reichsjustizgesetzen für die deutsche Nationenbildung zugeschrieben werden konnte - von einer gewissen Symbolwirkung als Konkretisierungen der Staatszielbestimmungen des Sozial- bzw. des Umweltstaats abgesehen. Eine im Blick auf jenen mythischen Maßstab heutzutage verbreitete Kodifikationsskepsis (S. 73ff.) beruft sich auf die Kompromisszwänge einer pluralistischen Demokratie und die Entscheidungsdefizite des Parlaments, auf den beschleunigten sozialen und technischen Wandel, auf die abweichenden Normstrukturen des Europarechts oder auf die Einbettung des Gesetzgebungsprozesses in die Stimmungsschwankungen der (Massen-)Mediendemokratie; indessen sind das letztlich keine Ausschlusskriterien, wie nicht nur erfolgreiche Kodifikationsprojekte (auch in Nachbarländern) und die Existenz vieler derartiger Vorbehalte seit über 100 Jahren zeigen (S. 94 f.). Vielmehr bleibt das Bedürfnis nach Kodifikationen (S. 88 f., 101ff.) wegen ihrer „Kodifikationsrendite" bestehen: durch die rationalisierende Kraft einer Systematisierung, die Entlastung für Rechtsanwender, die Erleichterung der Umsetzung europäischen Richtlinienrechts oder die Etablierung eines Rechtsgebiets. Bücherkodifikationen erscheinen als eine neue Form, die politischen Realisierungschancen eines Kodifikationsprojekts trotz der genannten Schwierigkeiten zu verbessern. Das Grundgesetz als Rabmen enthält im Blick auf Kodifikationen - anders als frühere Verfassungen zwar keine direkten oder indirekten Kodifikationsaufträge (S. 110ff.), wenn man von der unerfüllten „Verfassungserwartung “ eines UGB in Art. 125 b Abs. 1 S. 3 GG absieht (S. 116 f.); auf der anderen Seite kann es durch die Verteilung der Gesetzgebungskompetenzen bei Querschnittsmaterien Kodifikationen erschweren (S. 122 f.), zieht aber Bücherkodifikationen als solchen - bis zur Grenze eines Gesetzestorso keine Schranken.

b) Nach diesen eher allgemeinen grundlegenden Partien widmet sich die andere Hälfte der Arbeit ausgewählten Fragen der Gesetzestechnik bei Bücherkodifikationen. Auch wenn diese nach Maßgabe der ausgewählten Referenzen nicht den gleichen Grad der Systematisierung erreichen wie klassische Kodifikationen, so enthalten auch sie deren spezifische Techniken zur Vereinfachung und Rationalisierung. Diese werden jeweils beschrieben und typologisiert, in ihren Funktionen erläutert und im Blick auf Besonderheiten bei Bücherkodifikation diskutiert. Bei den Begriffsbestimmungen (S. 134ff.) werden explizite Definitionen von Kontextdefinitionen unterschieden; beide Erscheinungsformen vervollständigen und konkretisieren andere Normen und entfalten, insoweit ähnlich wie Prinzipien, Leitfunktion: Sie tragen - systematisch am Beginn des Gesetzes hervorgehoben - zum systematischen $\mathrm{Zu}$ sammenhalt der (ggf. auch noch nicht kodifizierten späteren) Gesetzesteile bei, verdeutlichen die innere Einheit eines Gesetzes („Integrationsfunktion“) und verkürzen dessen Umfang, wie am Beispiel nur des UGB-Entwurfes plausibel gemacht wird 
(S. 137ff.), doch unterliegen sie wegen der zeitlichen Streckung des Kodifikationsverfahrens der Gefahr von fehlerhaften Begriffsverwendungen (S. 146f.). Auch die Verweisungen (S. 148ff.) lassen sich typologisieren (deklaratorisch / konstitutiv; Außen- / Binnenverweisung; ausdrücklich / bezugnehmend u.a.); ihnen lassen sich ähnliche Funktionen wie bei den Begriffsbestimmungen zuordnen, doch gelten für die Bücherkodifikationen empirisch (nicht notwendig gesetzestechnisch) Systematisierungsdefizite, insoweit in UGB und SGB deutliche Verwendungsunterschiede bestehen (S. 152ff.). Rechtsprinzipien namentlich in Form von Ziel- und Zweckbestimmungen (S. 156ff.) sind trotz ihrer Ablehnung durch das offiziöse Handbuch der Rechtsförmlichkeit des Bundesministeriums der Justiz gängige Gesetzespraxis jedenfalls im SGB und im Entwurf eines UGB, weil auch sie den Rechtsanwender namentlich im Rahmen der teleologischen Auslegung unterstützen und „integrativ“ eine widerspruchsfreiere Gesetzesanwendung erleichtern; sie sollen zudem eine Informationsfunktion gegenüber dem Bürger haben und den Gesetzgeber bei späteren Kodifikationsschritten binden (können), sofern sie im jeweiligen Rechtsgebiet als Optimierungsgebote eine besondere Bedeutung haben (S. 163 f.).

Die zusammenfassende Diagnose einer gewissen Systematisierungsschwäche von Bücherkodifikationen durch einzelne Normtypen bestätigt sich für zusammenfassende Normgruppierungen wie die Allgemeinen Teile (S. 167ff.) - seien es solche im „systematischen“ Sinne (durch „Vor-die-Klammer-Ziehen“), seien es solche im „grundsätzlichen “ Sinne, d. h. in Form ausdrücklich formulierter Wert- und Richtungsentscheidungen des Gesetzgebers. Vor allem das SGB konnte noch nicht auf ein bestehendes Allgemeines Sozialrecht zurückgreifen, so dass sich allgemeine Regelungen auf mehrere Bücher verteilen (S. 36 f., 171ff.). Demgegenüber konnten sich die UGBEntwurfsverfasser auf eine langjährige wissenschaftliche Vorbereitung (vgl. S. 47ff., 182, 187) und ein funktional äquivalent wirkendes Allgemeines Umweltrecht aufgrund europarechtlicher Richtlinien-Vorgaben vor allem hinsichtlich des medienübergreifendes Ansatzes (vgl. auch S. 91, 186 f.) stützen. Folglich finden sich allgemeine Vorschriften im systematischen Sinne nur im ersten Buch des UGB I (ausf. S. 174ff.), ist die unterschiedliche Art der Verwendung von Allgemeinen Teilen nicht speziell der Technik einer Bücherkodifikation als solcher geschuldet. Freilich führt ihre zeitliche Streckung zu Modifikationen durch spätere Ergänzungen auch der allgemeinen Regelungen; das gilt unabhängig vom Umfang des Anwendungsbereichs eines Allgemeinen Teils - sei dieser ausdehnend durch eine „Integrationsklausel“ nach Vorbild des $\ 68$ SGB I auch schon für alle noch weiter bestehenden Fachgesetze festgelegt, sei er vor allem auf die bereits verabschiedeten Bücher beschränkt (wie beim UGB-Entwurf). Die äußere und innere Gesetzesstruktur insgesamt (S. 189ff.) wird entscheidend durch die Gründlichkeit eines Kodifikationsprogramms hinsichtlich dessen bestimmt, was positiv dem Regelungsanspruch der Kodifikation unterworfen wird und was negativ (z. B. in Rücksichtnahme auf andere Kodifikationen 
oder durch Ausschluss von Übergangsrecht) auszugrenzen ist; das erfordert gerade bei Querschnittsaufgaben wie im Sozial- und Umweltrecht schwierige Abgrenzungsentscheidungen. Ein solches Programm kann die Klarheit des Kodifikationsplans über die unterschiedliche Benennung und Anordnung der Bücher determinieren, ohne dass der parlamentarische Gesetzgeber in seiner späteren Gestaltungsfreiheit durch das Grundgesetz oder sonstige Gesetze (z.B. „Grundlagengesetze“) rechtlich für die Zukunft fest gebunden werden könnte. Die innere Struktur der Bücher von Bücherkodifikationen ähnelt stark der üblichen Gestaltung von einzelnen Fachgesetzen, wobei die Gestaltung der Übergangsvorschriften von entscheidender Bedeutung für die Akzeptanz der neuen Vorschriften sein soll (S. 133, 215). Demgegenüber sind Einführungsvorschriften (S. 217ff.) zur Änderung und Aufhebung anderer Vorschriften bei Bücherkodifikationen üblicherweise umfangreich und Ausdruck der spezifischen Funktion einer erfolgreichen Kodifikation, doch schafft die zeitliche Streckung zusätzliche Schwierigkeiten, ohne dass es wirkliche Alternativen zur Bücherkodifikation gibt (so nahe gelegt S. 229ff.).

3. Die Studie ist in mehrfacher Hinsicht ein Spiegel des Dilemmas der Gesetzgebungslehre in Deutschland. Das beginnt schon bei deren universitärer Verankerung als Wissenschaft. Schärdels Dissertation ist aus der juristischen Schwerpunktausbildung im Bereich „Rechtsgestaltung und Rechtspolitik“ an der Humboldt-Universität zu Berlin entstanden, die der Autor „als damals erster und einziger Studierender" absolvieren durfte (Vorwort, S. 5). Dahinter steht letztlich ein jahrzehntelanges spezielles Interesse seines Doktorvaters Michael Kloepfer, in dessen Umfeld nicht nur starke wissenschaftliche Impulse für den Erlass eines von ihm favorisierten UGB, sondern seit langem auch Dissertationen, in den letzten Jahren auch Habilitationsschriften zur Gesetzgebung entstanden sind; es gibt wohl überhaupt nur einen öffentlich-rechtlichen Lehrstuhl in Deutschland, der Gesetzgebungslehre in seiner Bezeichnung ausflaggt - den seines Schülers Matthias Rossi in Augsburg. Jenseits dessen ist eine akademische Gesetzgebungslehre nirgends nennenswert verankert, so sehr die „Neue Verwaltungsrechtswissenschaft“ (Andreas Voßkuble) die Rechtsetzungsorientierung stärken möchte. Es gehört wohl auch zu den Spätfolgen des staatsrechtlichen Positivismus und des von ihm dominierten rechtsdogmatischen Selbstverständnisses von Rechtswissenschaft, dass Gesetzgebungslehre als Forschungsfeld in Deutschland bis heute weithin brach liegt; selbst die „Zeitschrift für Gesetzgebung“ wird von rechtsdogmatischen Arbeiten zur Gesetzgebung, nicht von Arbeiten zur Gesetzgebungslehre im legistischen Sinne geprägt. ${ }^{1}$ Ganz anders findet sich etwa in der Schweiz ein deutlich höheres wissenschaftliches Niveau der Gesetzgebungslehre, das bei Schärdel symptomatischer Weise keine Resonanz findet. So muss er im Blick auf die wissenschaftlichen Erörterungen zur Gesetzgebungstechnik punktuell auf

1 Vgl. ausf. als Zeitschriftenrezension: H. Schulze-Fielitz, Zwanzig Jahre „Zeitschrift für Gesetzgebung“ als Seismograph der Gesetzgebungslehre in Deutschland, in: ZG 21 (2006), S. $209 \mathrm{ff}$. 
Thesen der 1970er Jahre zurückgreifen (vgl. z.B. S.169ff., zu Rödig und Kindermann), als namentlich von einem Kreis um den früh verstorbenen Jürgen Rödig wichtige Impulse zur Gesetzgebungslehre ausgingen, die aber heute wissenschaftlich weithin versandet sind. Ungeachtet der immer wieder über Deutschland verstreut entstehenden, oft historisch akzentuierten Dissertationen zur Gesetzgebung oder sonstiger Monographien ${ }^{2}$ als teilweise hervorragenden Einzelleistungen gibt es an den juristischen Fakultäten der Universitäten jenseits der Rechtsgeschichte keine wirkliche institutionell dauerhafte Schwerpunktbildung zur Gesetzgebungslehre die hier sichtbaren, sehr begrüßenswerten, institutionell neuen Berliner Bemühungen (auch mit eintägigen Tagungen und dem Start der neuen Schriftenreihe seit 2011, mit Schärdels Arbeit als erster Monographie) suchen dem wissenschaftlich abzuhelfen und so die mehr atmosphärisch wertvollen Veranstaltungen der Deutschen Gesellschaft für Gesetzgebung zu ergänzen.

4. Gibt es eine Nachfrage der Gesetzgebungspraxis nach einer akademischen Gesetzgebungslehre, wie sie etwa die Schweiz kennt? Es gehört zum „basso continuo“ in Schärdels Doktorschrift, die herausragende Bedeutung der Ministerialbürokratie für die Gesetzeserarbeitung im Vorfeld der parlamentarischen Beratungen stark zu betonen (vgl. z.B. S. 28, 81 f., 95ff.), abweichend von einer verbreiteten Fixierung auf das Parlament, das sich zur legistischen Feinarbeit ohne die Formulierungshilfen der Ministerialen auch in den Ausschussberatungen wenig eignet (vgl. auch S. 84). Warum aber kann die deutsche Ministerialbürokratie auf eine akademische Gesetzgebungslehre scheinbar ohne Verluste verzichten und sich mit praktisch ausgerichteten Fortbildungskursen an der eigenen Bundesakademie für öffentliche Verwaltung begnügen? Ein Grund dürften die Strukturen heutiger Gesetzgebung sein, die ganz dominierend dem Verwaltungsrecht, besonders dem Sozial- und Steuerrecht zuzuordnen sind (vgl. S. 100): Sie ändern weitestgehend bestehende Gesetze, deren Gerüste als Simile ohne aufwändige gesetzgebungstheoretische Vorüberlegungen genutzt und modifiziert fortgeschrieben werden können - die Grundfragen einer ganz neuen Kodifikation stellen sich nur ausnahmsweise, so dass dann zeitlich aufwändige Kommissionen vorbereitend tätig werden (vgl. S. 35, 47ff.). Den Rest besorgen das Handbuch der Rechtsförmlichkeit oder die fast zwei Dutzend auch mit der Prüfung der Rechtsförmlichkeit im Wege der Ressortabstimmung befassten Referate im Bundesministerium der Justiz. Speziell Probleme einer Bücherkodifikation stellen sich erst recht so selten, dass dem Beobachter außer SGB und UGB sonst nichts ins Auge fällt; manche Folgerungen Schärdels aus seinem Beispielsmaterial unterliegen daher der Gefahr einer Übergeneralisierung, etwa die These einer generell hervorgehobenen Rolle von Begriffsbestimmungen speziell bei Bücherkodifikationen (S. 137).

2 Vgl. jetzt G.F. Schuppert, Governance und Rechtsetzung. Grundfragen einer modernen Regelungswissenschaft, 2011. 
Die Dominanz der Ministerialbürokratie bei der Gesetzeserarbeitung hat auch eine Kehrseite, nämlich gegensätzliche institutionelle Eigeninteressen innerhalb der Bundesregierung (angesprochen z.B. S. 34, 57, 60, 96ff., 127) oder auch innerhalb eines Ministeriums (vgl. S. 56) oder zwischen Bundes- und Länderverwaltungen (vgl. S. 56 f.) mit negativen Folgen vor allem auch für das Projekt eines UGB, etwa wenn eine unterschiedliche Ressortzuständigkeit das Projekt einer Kodifikation insgesamt gefährdet (vgl. dazu S. 51, 53, 55, 96 f., 196 f., 200). Dabei geht es indessen oft um mehr als nur um institutionelle Eigeninteressen der Ministerialbürokratie; diese repräsentieren vielmehr ihrerseits fundamentale gesellschaftliche Interessen, deren Durchsetzungsmacht sie abbilden und ohne deren Berücksichtigung die Gesetzgebungslehre farblos bleiben muss. Es ist kein Zufall, dass in der Reputationshierarchie der Ministerien das Umweltministerium sehr weit unten, die klassischen Ministerien oben stehen, nicht nur aus Gründen der historischen Abfolge ihrer Entstehung, sondern als Ausdruck machtvoller und unterschiedlich durchsetzungsstarker Interessen in der Gesellschaft ${ }^{3}$ - diese Dimensionen der praktischen Wirksamkeitsbedingungen einer politikwissenschaftlich sensibilisierten Gesetzgebungslehre bleiben bei Schärdel ungeachtet des eigenen Anspruchs (S. 21) unterbelichtet. Gewiss werden solche Widerstände immer wieder angesprochen (in historischer Perspektive S. 30, strukturell S. 100 f.) und die Notwendigkeit und Normalität von Kompromissen betont (S. $75 \mathrm{ff}$., 105 f., 185), doch wird z.B. eine belastbare Erklärung, warum entgegen der jahrelang einmütigen Auffassung der Rechtswissenschaft die Auffassung des Bundesministeriums der Justiz zur fehlenden Bundeskompetenz für ein UGB sich 1999 durchgesetzt hat, sich kaum allein mit „Ressortegoismen“ als maßgeblichen Gründen (S. 53, 96) begnügen dürfen, so wie „fehlender politischer Wille“ $(\mathrm{S} .73,82)$ mehr eine Frage stellt als eine Antwort gibt.

5. Man fragt sich deshalb nach der Lektüre, warum die dargelegten Vorteile einer Bücherkodifikation nicht zum Erfolg geführt haben, trotz der vermeintlich „enormen symbolischen Wirkung für das Gemeinwesen“ (S. 236). Schärdel kann darauf keine Antwort geben, eben weil er die politischen, wirtschaftlichen und sozialen Interessen am Gesetzgebungsprojekt als Schranken für die normativen Ansprüche einer Gesetzgebungslehre zu sehr beiseite lässt (vgl. etwa S. 57, 69 f.): Das bayerische Veto gegenüber einer UGB-Kodifikation gründete wohl auch in der Furcht der Vertreter der organisierten Landwirtschaft in Bayern vor unkalkulierbaren Folgebelastungen - ihnen (und der Nähe einer bayerischen Staatsregierung zu ihrer Stammwählerschaft) lässt sich mit der Gesetzgebungstechnik der Bücherkodifikation nun einmal nicht entscheidend begegnen. Schon Schärdels nicht wirklich hinterfragte Prämisse einer „Kodifikationsrendite“ ist umstritten: Der eingespielte Status quo einer Gesetzeslage hat für die Praxis ein eigenständiges Gewicht (so auch S. 98), zumal wenn

3 Vgl. auch U. Smeddinck / R. Tils, Normgenese und Handlungslogiken in der Ministerialverwaltung, 2002, S. $236 \mathrm{ff}$. 
das Kodifikationsprojekt in seinem sachlichen Regelungsgehalt kaum etwas ändert. ${ }^{4}$ Die spezialisierten Verwaltungseinheiten sind eingespielt und mit „ihrem“ Gesetz zufrieden; demgegenüber haben kodifikationsästhetische Vorstellungen der Wissenschaft nur geringes Gewicht. Streckenweise liest sich die Darstellung von Schärdel eher wie eine immanente gesetzgebungstheoretische Rechtfertigungsschrift für eine Kodifikation des UGB.

Gesetzgebungslehre bleibt dabei auf einer vorgelagerten technischen Ebene stehen, für die Verständnis von Politik als einem sehr machtvollen Verteilungskampf um Ressourcen und Interessen fehlt und die auch die positivistische Trennung von Recht und Politik auf der Ebene der Rechtsetzung wiederholt. Politische Widerstände können dann eher als Bedrohung für den Vorteil von Bücherkodifikationen erscheinen, politische Vetospieler im Kodifikationsprozess zu reduzieren (so z.B. S. 186, s. auch 106). Das ist analytisch für eine juristische Dissertation im engeren Sinne sehr gut nachvollziehbar und aller Ehren wert, begrenzt aber den Erkenntniswert erheblich, wo nur ein interdisziplinäres Vorgehen die Angemessenheit gesetzgebungstheoretischer Folgerungen diskutieren könnte. Dahinter verbirgt sich das grundlegende Dilemma, das der wissenschaftliche Erkenntnisanspruch einer Gesetzgebungslehre in einer Demokratie nur eine dienende Funktion für den politischen Gesetzgeber haben kann. Gesetzgebungslehre müsste daher unter Verzicht auf „allein richtige“, implizit politisch (mit-)entscheidende gesetzestechnische Empfehlungen arbeiten können, die sich hinter Forderungen etwa nach „Vereinfachung “, „Modernisierung“ oder „Klarstellung" oft verbergen, aber eben auch hinter kodifikatorischer Formreinheit (vgl. S. 84), wenn man nicht rechtswissenschaftliche gegen politische Richtigkeit ausspielen will (vgl. S. 197, 241). Sie hätte selbst einen inhaltsneutralen Baukasten alternativer Gestaltungsformen für die unterschiedliche Vielfalt von politischen Gestaltungsansprüchen systematisch bereitzustellen, so dass ihre Empfehlungen vom Verdacht (krypto-)politischer Entscheidungsansprüche der Legistik-Experten völlig frei erscheinen. Von einer solchen Fähigkeit, geschweige von solchen Erkenntnisresultaten dürfte die Gesetzgebungslehre in Deutschland weit entfernt sein.

4 Vgl. K. Schönenbroicher / S. Gregor, Der Streit um das UGB I - Verfahrenskonzentrationen im Allgemeinen und Besonderen Verwaltungsrecht, NWVBl. 2009, S. 329 (330, 334ff.). 